\title{
Application Research of Multi-Software Collaboration Based on Complex Surface Design
}

\author{
Zhang Hua \\ Liaoning Economy Vocational Technological College, Shenyang, Liaoning, China, 110122
}

Keywords: bas-relief, innovative design, multi-software collaboration

\begin{abstract}
Bas-relief is widely used as it expresses people's yearning and good wishes to the future life, and gives people a sense of subtle elegance with its beautiful lines and color. Since it is hard for traditional bas-relief design to meet the demand of rapid developing science and technology, the research on using the collaboration of some different software to solve the problems and realize the seamless cohesion with the help of the respective special features of variety of CAD software and the compatibility of data interface has proved that the approach is a qualitative improvement because it can design a bas-relief work perfectly and transplant and graft the designed relief on other products.
\end{abstract}

\section{Introduction}

Bas-relief comes from the combination of sculpture and drawing, and uses the way of compression to deal with objects, and the factors like perspective to express the 3 direction space, with only one or two sides for people to watch $^{[1]}$. With the rising living standard, people wish to apply the bas-relief of good wishes and blessings to daily supplies and health care equipments. Owing to the substantial increase of demand and the rapid development of science and technology, automated production gradually replaced the handmade. At present, the widely used relief designing softwares in the market are mainly ArtCAM,Type3,JDPaint, all of which can turn graphic data of handpainted manuscript, scan documents, photos, grayscale into 3D relief digital model and generate code to drive numerical control machine to run.

However, it is hard for the structure designers to achieve the perfect combination of the elegant and complex relief structure and the inter-integrating product structure only with the relief software, so it must be achieved through CAD designing software of many majors. Thus, the quality requirement for designers is heightened, which requires designers to master various CAD software technologies and make their own designs integrated seamlessly, and how to realize the perfect combination of CAD software and relief software has become the key point to solve the problem.

\section{CAD Software and Relief Software}

CAD, the abbreviation of Computer Aided Design, refers to the use of computers and graphics devices to help designers to design. The wide application of CAD promotes the factories, enterprises and scientific research departments to improve technological innovation ability and speed up their product development.

Relief software, for example, ArtCAM can put graphic data of handpainted manuscript, scanning documents, photos, grayscale into a vivid and exquisite three-dimensional relief digital model, which will turn into more complex relief model through Boolean operation. The data format received by the software include: jpeg, art, 3da, 3ds, sldprt, igs and stl, and the output format as follows: the stl, obj, 3da, etc., among which the STL format is a three-dimensional relief output format that can store more intact data. The STL file format is a kind of 3d graphics file format mainly serving rapid prototyping manufacturing technology, and is composed of definition of multiple tri-patch ${ }^{[2]}$. The definition of each tri-patch include the 3d coordinate of each fixed point of the triangle and the method of vector of the tri-patch. Because the STL model only records the geometrical position information on the surface of the object without any expression of the topology information in relation to the relation among different geometries, so it will be a critical step to 
rebuild topology information and reconstruct entity model with relative position information. At present, commonly used CAD software such as Pro/E, UG, Catia can identify and receive the STL file, but can not directly edit and modify the file. Reverse design software such as Geomagic Studio, Imageware, CopyCAD, RapidForm can not only identify and receive the STL file, and edit and modify it, but also convert it to the 3d data format such as IGES, STP, DXF, DWG. However, this kind of software currently can only do the editing and modification on the base of the imported data, thus not so good as the 3d software such as UG, Pro/E, CATIA in terms of the structure designing. Even it can be realized, the dimensional accuracy and assembly accuracy can not meet the standard demand, and therefore, the edited data results need to be converted out and imported into other 3d software like UG, Pro/E, CATIA to redesign.

\section{Existing Problems in Relief Product Innovation and Design}

In this paper, a surface relief innovative design developed by a medical instrument factory is selected as an example. At present, Embossed graphic and characters are attached to all kinds of products to create beautiful performance effect, and the moral that relief itself presents is more popular. But our existing product surface relief is vacuous, lack of connotation and significance, making decorative relief tasteless and lack of cultural and artistic value. As shown in figure 1.

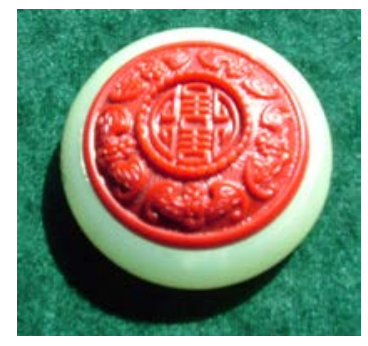

Figure 1 sample for reference

According to users' demand to apply the traditional Chinese auspicious decorating pattern "dragon and phoenix fly in extremely good fortune" to the decoration, among the variety of solutions drawn up after many sides collecting materials, as shown in figure 2, solution 5 is determined as the final plan through discussion.

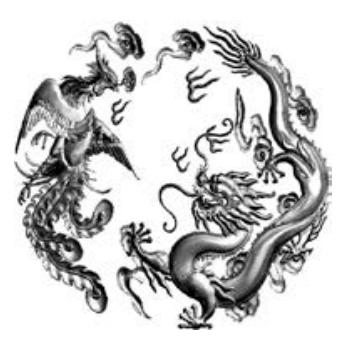

Solution 1

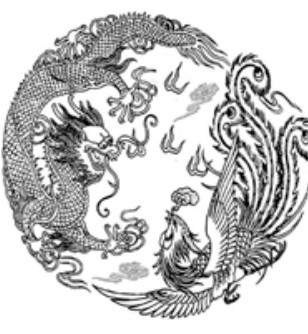

Solution2

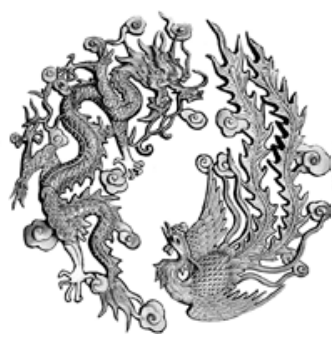

Solution 3

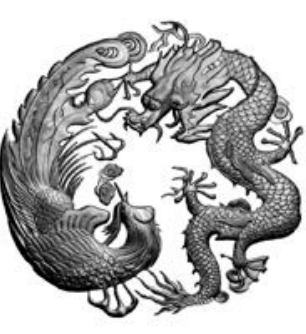

Solution 4

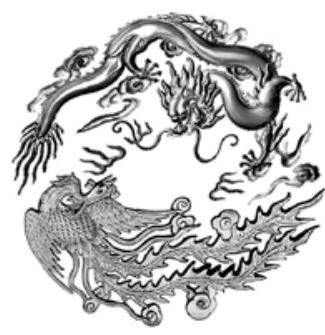

Solution 5

Figure 2 solutions drawn up

To quickly design out relief data model of the complex shape on product surface, we need to solve the following questions:

(1) CAD software is a powerful surface modeling function, but the product surface relief structure draft is exquisite with details of complex and irregular curve surface, which makes it hard to have surface modeling design directly with 3d CAD software, due to problems like trival design, large workload, slow speed of calculation and low efficiency ${ }^{[3]}$.

(2)Although the ArtCAM software can put the two-dimensional figure directly into three-dimensional relief, the precision, quality, color of the image itself and other factors will 
directly affect the generation effect of embossed relief. Because before ArtCAM software generates relief data, it will convert pictures to grayscale images, and then calculate the degree of concave and convex of the relief according to the depth of gray, the embossment effect is vulnerable to grayscale, considering that our pattern "dragon and phoenix fly in extremely good fortune" is very complex, .

(3) Although sculpting software ArtCAM can easily finish modeling of complex relief model, but there are big limitations to use it to design products, and data generated by sculpting software without NURBS properties can not interact free with the three dimensional software CAD.

\section{Multi-Software Collaboration to Realize the Scheme}

On the basis of the characteristics of 3d software like ArtCAM, Geomagic Studio, UG and data interface, we will generate the required bas-relief 3d data modeling. First we'll use ArtCAM to generate the $3 \mathrm{~d}$ data model (namely the bas-relief file), but the three-dimensional data model generated by this software can only be opened in the same kind of engraving software editor. Research has proven that the embossment file can be converted to STL triangular mesh, and the Geomagic Studio software can turn STL triangular mesh file into a three-dimensional entity or piece of data, and can reedit it to generate new data into the STP/IGES format, and modify or redesign it by using UG, CATIA or Pro/E 3d software.

(1) To use ArtCAM software feature to convert grayscale into relief data ${ }^{[4-5]}$ (2.5 dimensional geometry), we can simply complete the complex modeling of relief model. First, we need to use Photoshop software to adapt the product surface pattern to gray image, and adjust the contrast of the picture and drawing ratio, as shown in figure 3.

(2) To use ArtCAM software to turn the designed pattern of gray image into relief, as shown in figure 4, and then export the STL file.

(3) To import the STL file into the Geomagic Studio software, (processed data has NURBS properties) and then export it into the STP file format, as shown in figure 5.

(4) To import the STP file into UG software to merge the relief model into the surface of the product together to generate entity files and finish detail construction (the final product $3 \mathrm{~d}$ mathematical model is shown in figure 6). In UG software, 'surface/stitching' command will automatically stitch closed surface to generate entity, and those unclosed surface can also be added some thickness to generate entity, so it is very convenient in application.

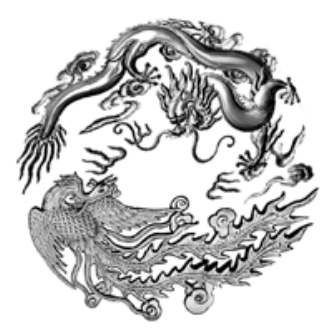

Figure 3

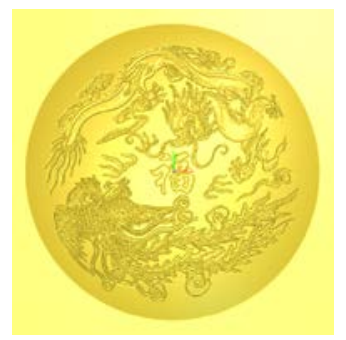

Figure 4

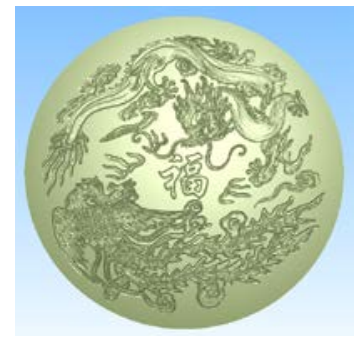

Figure 5

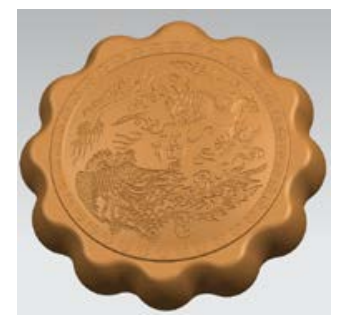

Figure 6

\section{Conclusion}

It has been verified through experiments that the use of the respective characteristics of different software and the data interface compatibility can help us completely realize the collaborative work of various software, and complete the development, innovation and design of a variety of complex products, and it can be applied to other fields to realize the collaboration of different departments and industries and to reduce the work intensity of the designer as well as the skill requirements, so it will enjoy wide application and popularity in the related fields. 


\section{References}

[1] Fang Zhongming: Bas-Relief Class Tutorial (Tianjin People's Fine Arts Publishing House,Chin a 2007)

[2]Jin tao, Tong Shuiguang: Reverse Engineering Technology (Mechanical Industry Publishing Ho use, China 2003)

[3] You Jia,Jiang Xianfeng:TheStudy on Design Technology of the Product Surface Relief,submitte d to Journal of Machine Tools and Hydraulic,vol. (2009),p.215-217.

[4] Kaiyan Yan,Hui Huang: Research of numerical control Engraving Technology Based on Dingital

Image, submitted to the Journal of Computer Application Research,Vol.24(8)(2007), p.206-208.

[5]Yongqiang Zhu,Congda Lu:Review on the Free Curveand Surface Modeling Technology,submitt ed to the journal of Chinese Manufacturing Informatization,Vol.32(5)(2003), p.110-113 